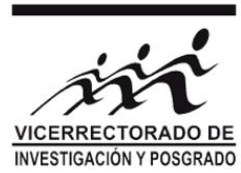

\title{
Cálculo de transferencia de esfuerzos estáticos para sismos de gran magnitud (Mw $\geq 8.0)$ ocurridos en la región Central del Perú desde 1940 al 2007
}

\author{
Nick Moreno*1,2 ${ }^{*}$ J. C. Villegas-Lanza ${ }^{2}$ y César Jiménez ${ }^{1}$ \\ ${ }^{1}$ Universidad Nacional Mayor de San Marcos, Lima-Perú \\ ${ }^{2}$ Instituto Geofísico del Perú. Unidad de Geodesia Espacial, Lima-Perú
}

Recibido 12 mayo 2019 - Aceptado 01 agosto 2019

\begin{abstract}
Resumen
En esta investigación hemos evaluado, analizado y mapeado el cambio y la transferencia de esfuerzos producidos por los grandes sismos ( $\mathrm{Mw} \geq 8.0$ ) ocurridos en la zona central de subducción de Perú durante los últimos 80 años. Los sismos que hemos analizado ocurrieron el 24 de mayo de 1940 ( $8.0 \mathrm{Mw}) ; 17 \mathrm{de}$ octubre de 1966 (8.1 Mw); 3 de octubre de 1974 (8.1 Mw) y 15 de agosto de 2007 (8.0 Mw). Los resultados muestran áreas donde el esfuerzo ha aumentado o disminuido. Para el evento sísmico de 1940 (8.0 Mw), la propagación del aumento de los patrones lobulares de transferencia de esfuerzos de Coulomb al norte de la ciudad de Huarmey ha generado valores de 0.568 bar que implicaron una menor activación de la sismicidad, debido a la antigüedad del sismo no existe una buena correlación de réplicas. Para el evento sísmico de 1966 (8.1 Mw), se observó que la zona norte cerca de la costa de Huarmey seguía cargada de esfuerzos porque la transferencia de esfuerzos de Coulomb tomó un valor de 0.382 bar, este resultado define una activación sísmica cerca de la costa de las ciudades de Huacho y Huarmey hacia el norte a la ciudad de Chimbote y hacia el sur a la ciudad de Lima, la correlación espacial de las réplicas es considerable en la dirección de la fosa peruana. Para el evento sísmico de 1974 (8.1 Mw), la ciudad de Huacho tiene una concentración de esfuerzos de 0.144 bares, la ciudad de Lima tiene una concentración de esfuerzos de -4.187 bares, la ciudad de Pisco tiene una concentración de esfuerzos de 0.140 bares. Además, la correlación espacial de las réplicas es buena en dirección sur cerca de la latitud $-14^{\circ}$ entre la fosa peruana y las costas de Pisco. Para el reciente sismo de Pisco en 2007 (8.0 Mw) encontramos una buena correlación entre los patrones lobulares positivos que indican esfuerzos transferidos y la ocurrencia masiva de réplicas en el área que limita el área de ruptura sísmica con valores de esfuerzos que varían entre 1.2 y 1.5 bares, lo que implica que se ha transferido una gran cantidad de esfuerzo estático para generar la secuencia de réplicas. Sin embargo, los esfuerzos ha aumentado en las costas de la ciudad de Cañete y en la zona costera del sur de Lima y esto podría generar un futuro sismo.
\end{abstract}

Palabras clave: Transferencia de esfuerzos, acumulación de esfuerzos, relajación de esfuerzos.

\section{Calculation of static stress transfer of large magnitude earthquakes (Mw $\geq 8.0)$ occurred in the central region of Peru from 1940 to 2007}

${ }^{*}$ nick.moreno@unmsm.edu.pe 


\begin{abstract}
In this research we have assesed, analized and mapped the change and stress transfer produced by the large earthquakes $(\mathrm{Mw} \geq 8.0)$ ocurred in the central Peru subduction zone during the last 80 years. The earthquakes that we have analyzed ocurred on May 24, 1940 (Mw 8.0); October 17, 1966 (Mw 8.1); October 3, 1974 (Mw 8.1) and August 15, 2007 (Mw 8.0). The results show areas where stress have increased and/or decreased. For the seismic event of 1940 (Mw 8.0), the propagation of increased lobular stress transfer patterns from Coulomb to the north of the city of Huarmey has generated values of 0.568 bar which implied a lower seismicity activation, due to the antiquity of the earthquake it does not have a good correlation of aftershocks. For the 1966 seismic event (Mw 8.1), it was observed that the northern area near the coast of Huarmey remained loaded with stresses because Coulomb's stress transfer took a value of 0.382 bar, this result defines a seismic activation near the coast of the cities of Huacho and Huarmey northbound to the city of Chimbote and southbound to the city of Lima, the spatial correlation of the aftershocks is considerable in the direction of the Peruvian trench. For the 1974 seismic event (8.1 Mw), the city of Huacho has a stress concentration of 0.144 bars, the city of Lima has a stress concentration of -4.187 bars, the city of $\mathrm{Pisco}$ has a stress concentration of 0.140 bars. In addition, the spatial correlation of the aftershocks is good in a southernly direction near latitude $-14^{\circ}$ between the peruvian trench and the coasts of Pisco. For the recent 2007 (Mw 8.0) Pisco earthquake we found a good correlation between the positive lobular patterns that indicate stress transferred and the massive occurrence of aftershocks in the area bounding the seismic rupture area with stress values ranging between 1.2 to 1.5 bars, which implies that a large amount of static stress has been transferred to generate the aftershock sequence. However, stress has increased on the coasts of the city of Cañete and the southern coastal area of Lima and this could generate a future earthquake.
\end{abstract}

Keywords: Stress transfer, strain accumulation, stress relaxation.

\section{Introducción}

La sismicidad del Perú tiene su punto de partida en el proceso de subducción, que consiste en la subducción de la Placa de Nazca debajo de la Placa Continental a una velocidad de convergencia relativa de 6-7 cm/año en la Región Central del Perú; Esta región está limitada por la zona de fractura de Mendaña (MFZ) $\left(\sim 10^{\circ}\right)$ al norte y la cresta de Nazca $\left(\sim 15^{\circ}\right)$ al sur, y se caracteriza por la ocurrencia de grandes sismos ( $\mathrm{Mw} \geq 8.0$ ) cuya recurrencia es alrededor de 100-300 años (Kelleher, 1972 [Kel72]; Dorbath et al., 1990 [Dor90]; Sladen et al., 2010; Villegas-Lanza et al., 2016) (Figura 1). Para comprender el proceso de subducción a lo largo de la costa de Preuvian, varios investigadores (Barazangui e Isacks, 1979 [Bar79]; Hasegawa e Isacks, 1981 |Has81]; Schneider y Sacks, 1987; Rodríguez y Tavera 1991 [Rod91]; Cahill e Isacks, 1992 [Cah92]; Tavera y Buforn, 1998) concluyeron que este proceso es heterogéneo y ocurre en dos formas, una es la subducción normal (en la región sur del Perú) y la otra subducción sub-horizontal (en la región central y norte del Perú), cada una de las cuales es función del epicentro y la ubicación de la profundidad focal de el evento sísmico, y ambos producen una contorsión entre las placas tectónicas. La Región Central del Perú presenta una subducción sub-horizontal o plana y su peculiaridad es que comienza en un ángulo de $12^{\circ}-15^{\circ}$ para alcanzar una profundidad promedio de 120 $\mathrm{km}$, lo que implicaría que la placa se movería horizontalmente hasta $500 \mathrm{~km}$ desde el punto de partida de la línea de trincheras (Tavera y Buforn, 1998 [Tav98]; Villegas-
Lanza et al., 2016 [Vil16] ).

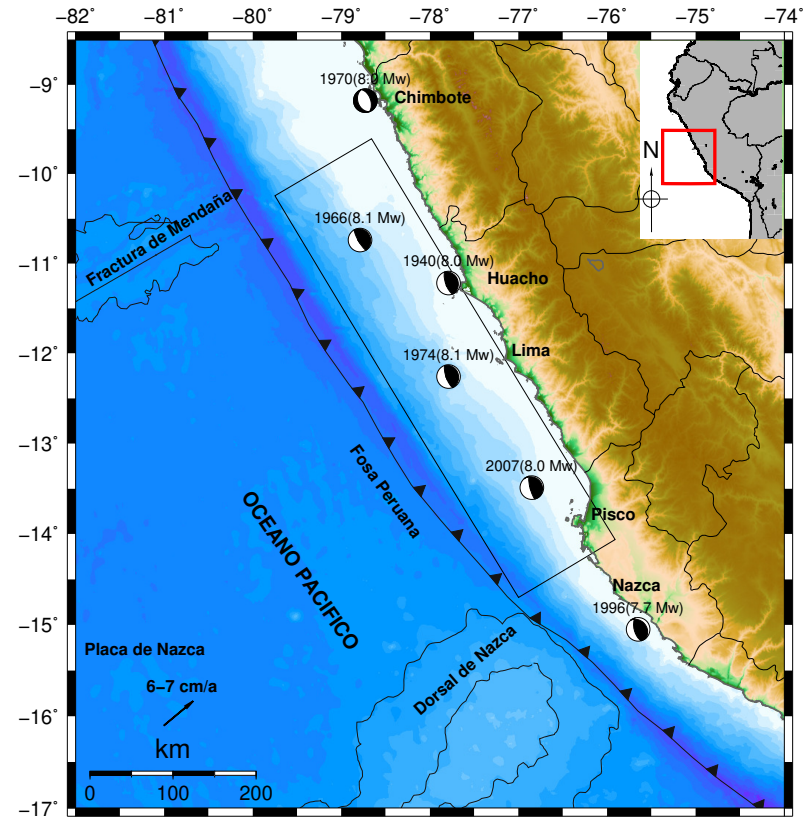

Figura 1: Escenario sismotectónico del área de estudio correspondiente a la zona de subducción de la región central del Perú. Los epicentros de los sismos principales están representados por diagramas focales, el rectángulo negro representa el área de ruptura aproximada del gran sismo de $1746(\sim 8.8 \mathrm{Mw}-9.0 \mathrm{Mw})$ como fue propuesto por Jiménez et al. (2013). 
Durante más de 273 años después del gran sismo de subducción de 1746 (8.8-9.0 Mw) (Jiménez et al., 2013) [Jim13] ocurrió en la región central de Perú, el área entre la Zona de Fractura de Mendaña y la Cordillera de Nazca sufrió 4 sismos del Gran magnitud igual o mayor que 8.0 Mw en 1940, 1966, 1974 y 2007. Además, hay 2 eventos sísmicos de magnitud similar ubicados cerca de la Zona de Fractura de Mendaña y la Cordillera de Nazca ocurridos en 1970 y 1996, respectivamente, que podrían estar relacionados con los primeros (Figura 1 ).

Desde el evento de 1746 (8.8-9.0 Mw), no se ha producido un evento de magnitud similar, y aunque las áreas de ruptura de la secuencia de eventos de los últimos 80 años cubren espacialmente toda el área de ruptura del gran sismo de 1746, el balance del momento sísmico sugiere que la secuencia de eventos en los últimos 80 años habría liberado parcialmente los esfuerzos acumulados, esto es alrededor de una cuarta parte del momento sísmico de 1746 (Villegas-Lanza et al., 2016 [Vil16]; Chlieh et al., 2011 [Chl11]; Jiménez et al., 2016 [Jim16]). Es por eso que la preocupación de esta investigación surge al saber cuál es la contribución en la relajación y transferencia de esfuerzos de 4 sismos que ocurrieron en la zona de subducción de la región central del Perú, evaluando las zonas de máximo aumento de esfuerzos y su correlación con la sismicidad actual y su equilibrio con respecto al momento sísmico lanzado por el gran evento de 1746.

Una de las metodologías que permite el cálculo de la transferencia y los cambios de esfuerzos causados por un evento o un conjunto de eventos sísmicos es la propuesta por Toda et al. (2011) en el software Coulomb 3.3. En todo el mundo, el uso del cambio de esfuerzos de Coulomb $(\triangle C F C)$ se ha estudiado más en el sismo de Landers (Estados Unidos) en 1992, cuya magnitud fue 7.4 Mw (King et al., 1994), también en Japón, por Toda et al. (2011) [Tod11] para el sismo de Kobe cuya magnitud fue 6.9 Mw. En zonas de subducción, el software Coulomb 3.3 ha sido utilizado en la región de Coquimbo (Chile) por Gardi et al. (2006) donde ocurrieron 12 sismos con una magnitud entre 6.0 Mw y 7.6 Mw en 1997. En Ecuador, Mayorga y Sánchez (2016) lo estudiaron para el sismo de Esmeraldas en 1906 de magnitud 8.8 Mw. En Perú solo hay un resumen de investigación de Robinson et al. (2001) [Rob01], quienes estudiaron el sismo de Arequipa de 2001, cuya magnitud fue de 8.4 Mw. Todos ellos muestran resultados de patrones lobulares y zonas de activación sísmica.

El objetivo de esta investigación es estudiar, analizar y mapear la transferencia y los cambios de esfuerzos producidos por eventos de magnitud $\mathrm{Mw} \geq 8.0$ ocurridos entre 1940 y 2007 . Los resultados permiten identificar las áreas de mayor y menor transferencia de esfuerzos producidos por aquellos eventos.

\section{Datos}

Los parámetros hipocentrales se obtuvieron de los mecanismos focales de cada evento sísmico a gran escala $(\mathrm{Mw} \geq 8.0)$ de la zona de subducción de la región central del Perú. Para esta compilación, hemos utilizado la información en catálogos sismológicos como el NEIC (Centro Nacional de Información sobre sismos) del Servicio Geológico de los Estados Unidos, también del sitio web del Global CMT (Global Centroid Moment Tensor) y, finalmente, estudios de investigación citados en las referencias Los parámetros del mecanismo focal de cada evento sísmico se enumeran en la Tabla 1.

\begin{tabular}{cccc}
\hline Evento & Strike $\left(^{\circ}\right)$ & $\operatorname{Dip}\left(^{\circ}\right)$ & Rake $\left(^{\circ}\right)$ \\
\hline 1940 & 340 & 20 & 90 \\
1966 & 335 & 12 & 90 \\
1974 & 340 & 17 & 90 \\
2007 & 318 & 20 & 63 \\
\hline Mw & Prof $(\mathrm{km})$ & Epicentro & Mo $(\mathrm{Nm})$ \\
\hline 8.0 & 30 & $-11.22 /-77.79$ & $2.5 \times 10^{20}$ \\
8.1 & 21 & $-10.74 /-78.79$ & $20 \times 10^{20}$ \\
8.0 & 13 & $-12.39 /-77.66$ & $15 \times 10^{20}$ \\
8.0 & 18 & $-13.49 /-76.85$ & $12 \times 10^{20}$
\end{tabular}

Tabla 1: Parámetros del mecanismo focal de cada evento sísmico (Beck y Ruff, 1989; Sladen et al., 2010).

\section{Metodología}

Teniendo en cuenta que la Tierra puede representarse como un medio homogéneo, isotrópico, elástico y de medio espacio, el desplazamiento interno y el campo de deformación se obtienen utilizando un modelo de desplazamiento elástico basado en las ecuaciones analíticas de Okada (1992), que permite Calcular los cambios en el esfuerzo estático y la deformación generados por la ruptura de un sismo (Freed, 2005; Stein, 1999; King et al., 1994; Miao y Zhu, 2012 [Mia12]).

En el criterio de Coulomb, la falla ocurre cuando en un plano los esfuerzos de Coulomb $\sigma_{f}$ excede un valor específico (Freed, 2005; Stein, 1999; King et al., 1994; Miao y Zhu, 2012):

$$
\sigma_{f}=\tau-\mu\left(\sigma_{n}+P\right),
$$

Donde $\tau$ es el esfuerzo cortante en el plano de falla, $\sigma_{n}$ el esfuerzo normal, $\mathrm{P}$ la presión del fluido poroso y $m u$ el coeficiente de fricción. Dado que es difícil medir directamente el valor del esfuerzo cortical real, la transferencia de esfuerzos de Coulomb se usa para describir el cambio relativo en el esfuerzo (Freed, 2005; King et al., 1994; Miao y Zhu, 2012). Cuando el coeficiente de fricción es constante, la ecuación (1) puede reescribirse como:

$$
\Delta \sigma_{f}=\Delta \tau-\mu\left(\Delta \sigma_{n}+\Delta P\right),
$$


Donde $\Delta$ es el cambio del parámetro correspondiente. La presión del fluido poroso $\mathrm{P}$ modifica el esfuerzo normal efectiva en el plano de falla. Cuando el esfuerzo de la roca cambia más rápidamente que la difusión de la presión del fluido, $\mathrm{P}$ puede estar relacionado con el esfuerzo de confinamiento por el coeficiente (B) de Skemptons, donde el coeficiente efectivo de fricción se define por $\mu^{\prime}=\mu$ (1-B). Este coeficiente $\left(\mu^{\prime}\right)$ puede explicar el comportamiento del fluido de los poros y la propiedad del plano de falla, en el rango 0-1 (Freed, 2005 |Fre05]; King et al., 1994; Miao y Zhu, 2012 [Mia12]). Entonces, la ecuación (2) puede reescribirse como:

$$
\Delta \sigma_{f}=\Delta \tau-\mu^{\prime}\left(\Delta \sigma_{n}\right)
$$

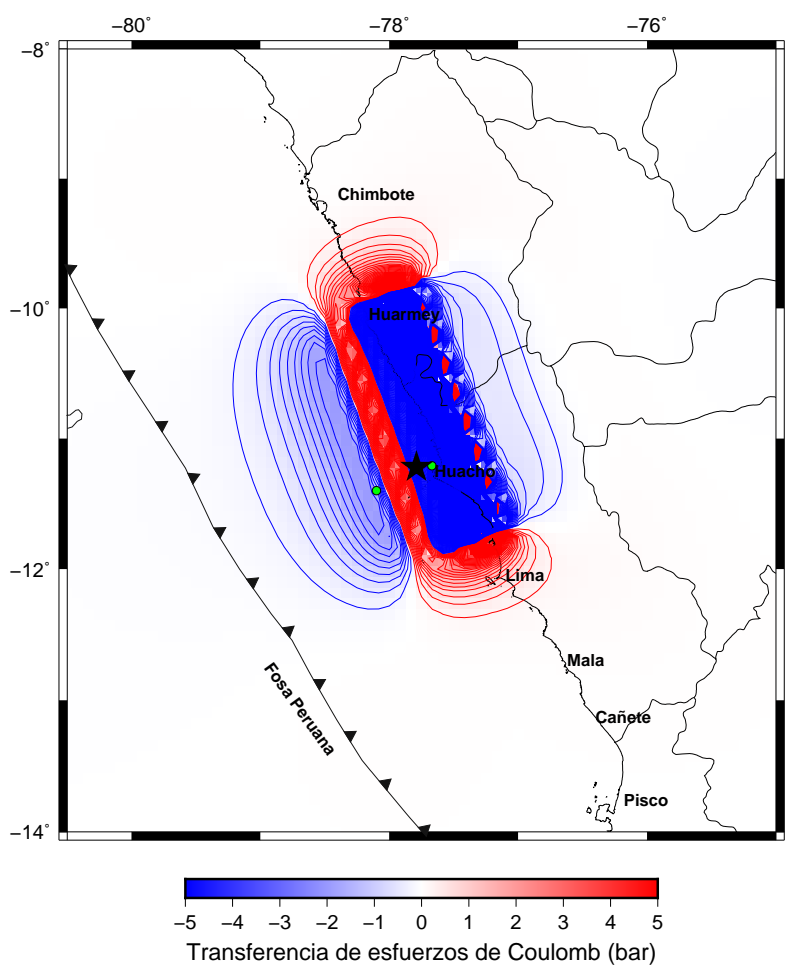

Figura 2: Modelado de la transferencia de esfuerzos de Coulomb del mecanismo focal propuesto por Beck y Ruff (1989) para el sismo de 1940. Los círculos verdes representan las réplicas, que se tomaron hasta una semana después del sismo principal. La estrella negra indica la ubicación del epicentro. Los contornos rojos indican un aumento de esfuerzo de 0.2 bar y los contornos azules indican una caída de esfuerzos de 0.2 bar.

Donde el esfuerzo compresivo es positivo. El cambio de esfuerzo de Coulomb ( $\Delta$ CFS) se define en el plano de falla específico, que se llama falla de recepción; esto promueve la ocurrencia de sismos cuando $\Delta \sigma_{f}>0$. Aunque el $\Delta$ CFS causado por un sismo es considerablemente menor que el esfuerzo acumulado requerido para un sismo, muchas actividades sísmicas muestran que el cambio de esfuerzos de Coulomb de más de 0.1 bar parece ser suficiente para provocar sismos (Stein, 1999; King et al.1994; Miao y Zhu, 2012 [Mia12]).

\section{Cálculo de $\triangle$ CFS para la secuencia de sismos de 1940 a 2007}

Se mencionan los principales resultados de la secuencia sísmica de 1940, 1966, 1974 y 2007 :

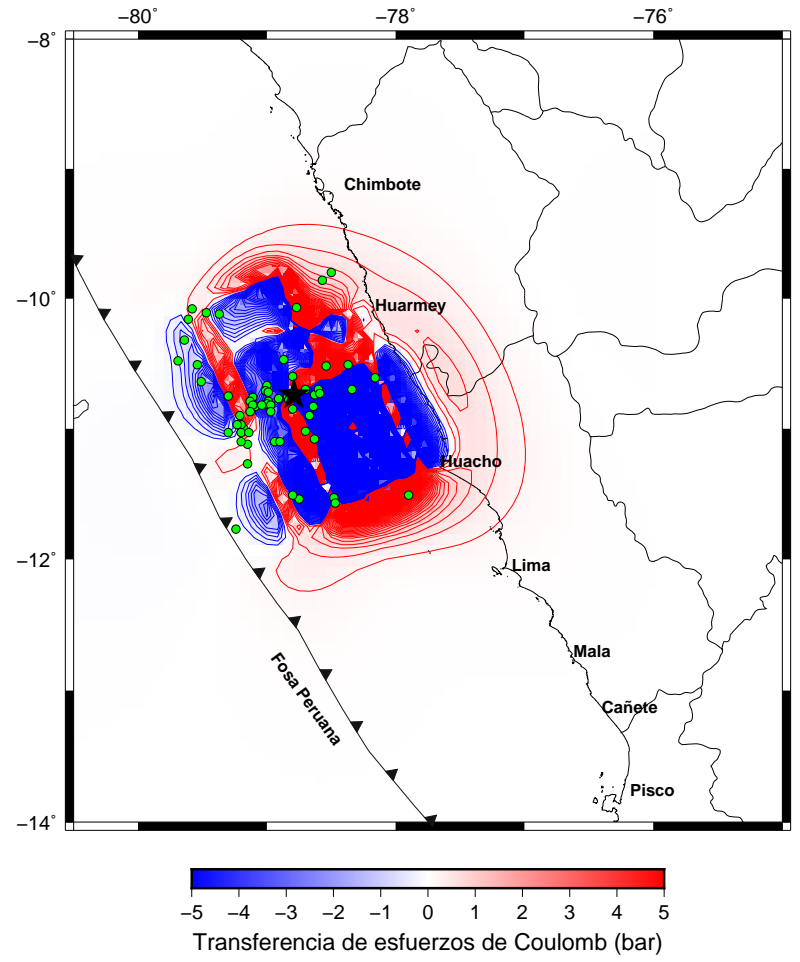

Figura 3: Modelado de la transferencia de esfuerzos de Coulomb del mecanismo focal propuesto por Beck y Ruff (1989) para el sismo de 1966. Los círculos verdes representan las réplicas, que se tomaron hasta una semana después del sismo principal. La estrella negra indica la ubicación del epicentro. Los contornos rojos indican un aumento de esfuerzo de 0.2 bar y los contornos azules indican una caída de esfuerzos de 0.2 bar.

Para el evento de 1940 (Figura 2), la transferencia de esfuerzo de Coulomb exhibe un aumento y propagación de los patrones lobulares que se extienden a las costas de Huarmey, más al norte, estos patrones lobulares se atenúan hacia el mar y hacia el continente. En el puerto de Huarmey, el valor de $\triangle$ CFS era de 0,568 bar, lo que implicaba una menor activación de la sismicidad de la zona y sus alrededores. En la ciudad costera de Huacho, el valor de transferencia de esfuerzos fue de -9.614 bar, lo que implicó una relajación de los esfuerzos. Los patrones lo- 
bulares de transferencia de las esfuerzos de Coulomb se extendieron hacia el sur hasta la ciudad de Lima tomando valores de 0.367 bar, lo que implicaba una activación de la sismicidad más baja porque podría ser un límite de propagación de ruptura; más al sur, estos lóbulos se atenúan en dirección a la fosa peruana y al continente.

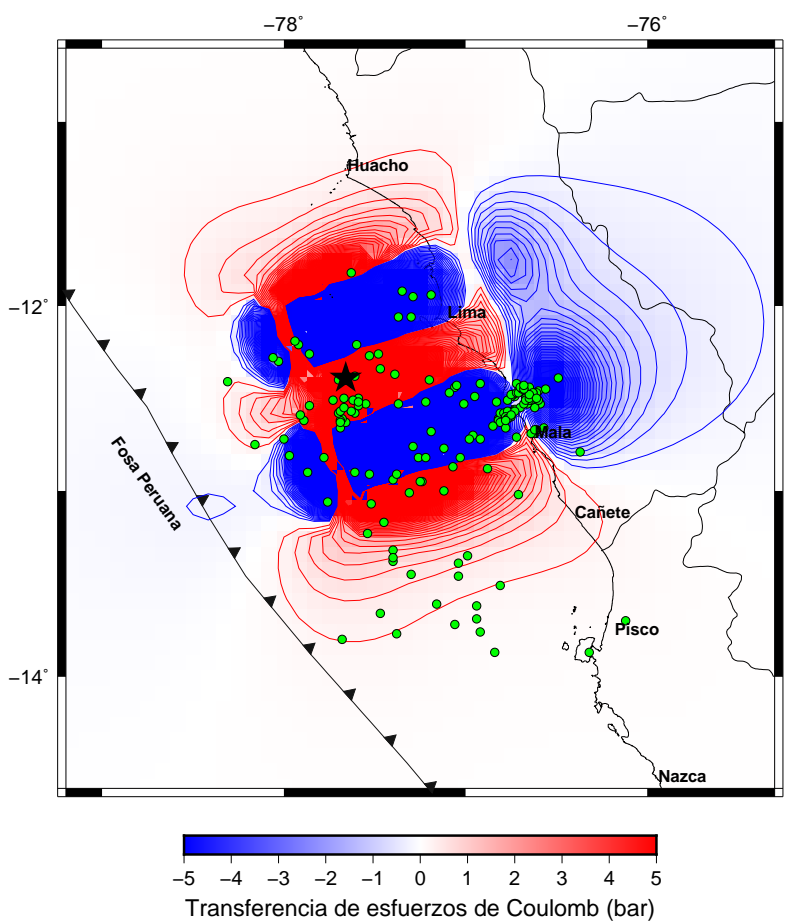

Figura 4: Modelado de la transferencia de esfuerzos de Coulomb del mecanismo focal propuesto por Beck y Ruff (1989) para el sismo de 1974. Los círculos verdes representan las réplicas, que se tomaron hasta una semana después del sismo principal. La estrella negra indica la ubicación del epicentro. Los contornos rojos indican un aumento de esfuerzo de 0.2 bar y los contornos azules indican una caída de esfuerzos de 0.2 bar.

Para el evento de 1966 (Figura 3), Jiménez et al. (2016) [Jim16] concluye que el máximo deslizamiento o dislocación se encuentra en el área sur del plano de ruptura, en alta mar en dirección a la ciudad de Huacho; de hecho, esta área es la mayor área de liberación de energía corroborada por el modelo (Figura 3). La transferencia de esfuerzoss de Coulomb en la costa de Huarmey tomó el valor de 0.322 bar, lo que implicó una menor inhibición del cociente de fricción deslizante y, por lo tanto, el esfuerzo normal tomó el valor de -0.017 bar, este pequeño valor de esfuerzo confirma un acoplamiento débil de la zona y, como resultado, una pequeña variación de los valores de cambio de esfuerzos de Coulomb ( $\triangle$ CFS) con el componente de corte. Cuando se discretiza la fuente sísmica, notamos que la zona norte cerca de la costa de Huarmey permaneció cargada de esfuerzos porque la transferencia de esfuerzos de Coulomb tomó un valor de 0.382 bar, este resultado define una activación de la sismicidad cerca de la costa de la ciudad de Huacho y Huarmey, limitada entre la fosa peruana y la costa.

Para el evento de 1974 (Figura 4), los lóbulos de acumulación de esfuerzos se extienden a los lados norte y sur de la geometría de la fuente sísmica. La bahía de Pisco y sus alrededores tiene una gran acumulación de esfuerzos, así como las costas de Huacho. Los lóbulos de relajación de esfueros están en dirección al continente y a la trinchera. La ciudad de Huacho tiene una concentración de esfuerzos de 0.144 bares, la ciudad de Lima y Callao tiene una concentración de esfuerzos de -4.187 bares, la ciudad de Cañete tiene una concentración de esfuerzos de -0.241 bares, la ciudad de Pisco tiene una concentración de esfuerzos de 0.140 bares y una activación de la sismicidad cerca de sus costas.

Para el modelo propuesto por Sladen et al. (2010) del evento sísmico de 2007, el plano de falla ha sido discretizado en 12 subfaults (Figura 5), la relajación de los esfuerzos se mantiene con mayor amplitud en la dirección del continente. En la ciudad de Pisco hemos calculado una transferencia de esfuerzos de -16.527 bar (Figura 5). Este resultado implicó una alta relajación de los esfuerzos en la sub-falla donde se encontraba la ciudad de Pisco y un alto acoplamiento. En la ciudad de Cañete hemos obtenido un valor de cambio de esfuerzos de Coulomb ( $\Delta$ CFS) de $-0,478$ bar, lo que implica una relajación de los esfuerzos y un valor promedio de acoplamiento sísmico, además de una menor activación de la sismicidad.

\section{Discusión}

La transferencia de esfuerzos de Coulomb se calculó y proyectó para los eventos sísmicos de $\mathrm{Mw} \geq 8.0$ ocurridos en la zona de subducción de la región central del Perú. Los cálculos se muestran de acuerdo con la profundidad hipocentral de cada evento sísmico. Esto ayudará a observar dónde se transfirió los esfuerzos y si estos no se relajaron con las réplicas, ya que estas áreas pueden tener un alto potencial para generar sismos importantes en el futuro.

\section{Evento sísmico de $1940(8.0 \mathrm{Mw})$}

Notamos en la Figura 2 que tres réplicas están ubicadas cerca del sismo principal (las costas de la ciudad de Huacho) pero coinciden dentro de la zona de relajación de esfuerzos (zona azul), las réplicas de todo el año fueron tomadas del evento sísmico, pero son de distribución irregular. A partir de este hecho, concluimos que la correlación entre el patrón de transferencia de esfuerzos de Coulomb y la distribución irregular de las pocas réplicas no es significativa. Debido al aumento de los esfuerzos del evento sísmico de 1940, se observa en la zona noroeste, el evento sísmico de 1966 podría haberse descartado (una 
secuencia de réplicas importantes o la energía no se liberó y todavía se puede liberar hasta la fecha) la relajación de esfuerzos concentradas en esa área y la migración de esfuerzos en el área sureste por la misma o acumulada para eventos sísmicos posteriores.

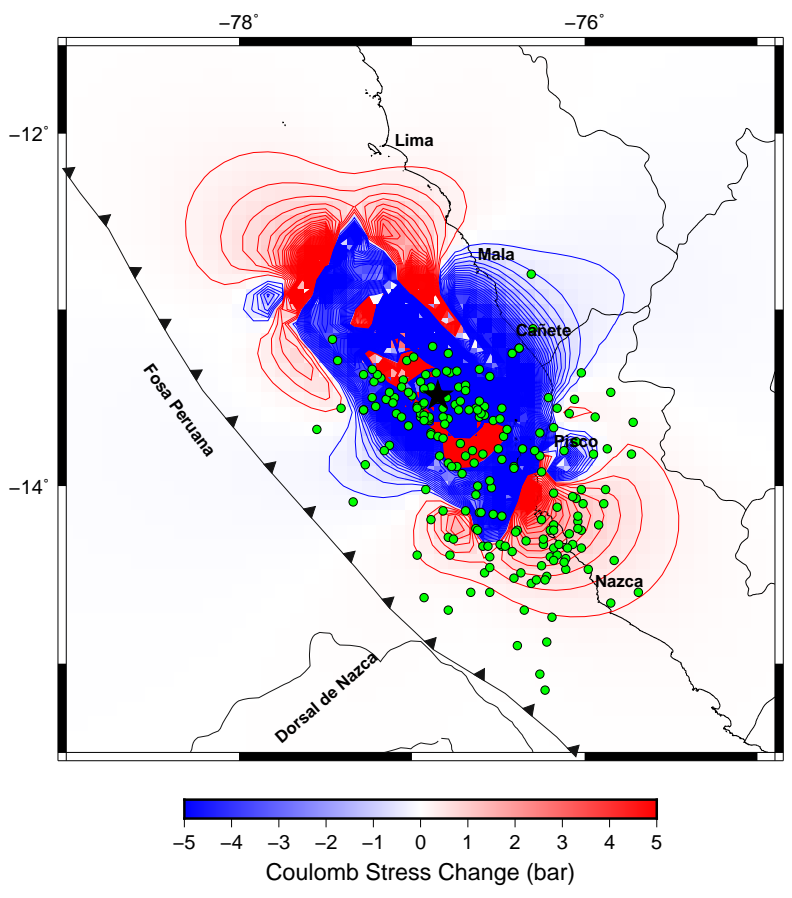

Figura 5: Modelado de la transferencia de esfuerzos de Coulomb del mecanismo focal propuesto por Sladen et al. (2010) para el sismo de 2007. Los círculos verdes representan las réplicas, que se tomaron hasta una semana después del sismo principal. La estrella negra indica la ubicación del epicentro. Los contornos rojos indican un aumento de esfuerzo de 0.2 bar y los contornos azules indican una caída de esfuerzos de 0.2 bar.

\section{Evento sísmico de 1966 (8.1 Mw)}

En la Figura 3, la distribución de las réplicas se observa principalmente (más del $50 \%$ ) al este o al oeste del área de ruptura. Al igual que el evento de 1940, no hay muchas réplicas que ayuden a una correlación óptima, según Stein (1999) [Ste99], un alto porcentaje de réplicas en áreas de mayor esfuerzos conduce a un buen efecto de un desencadenante de sismo en nuestra área de estudio. Este argumento lleva a la conclusión de que no hay réplicas en esta región, por lo que hoy habrá una gran acumulación de esfuerzos. Se observan algunas réplicas en dirección suroeste y el aumento de esfuerzos se dirige hacia las costas de Lima-Callao. La relajación de los grandes esfuerzos causadas por los sismos, como 1940 y 1966, no difieren significativamente de una región a otra. El acoplamiento en la zona de subducción para el sismo de 1966 no es tan débil y esto se observa en la Figura 3.

\section{Evento sísmico de 1974 (8.1 Mw)}

En la Figura 4, se observa una mejor correlación entre los patrones de esfuerzo lobular y las réplicas en comparación con los eventos sísmicos anteriores, esto se debe a un mejor valor de los patrones de dislocación. La mayor concentración de réplicas se encuentra al sur de la ciudad de Lima, entre las latitudes $-12^{\circ}$ y $-13^{\circ}$ cerca de la línea de fosa. La existencia de un aumento de los esfuerzos en el lado sureste del plano de falla indica que hay un aumento ubicado cerca de la fosa peruana y entre las latitudes $-13.5^{\circ}$ y $-14.5^{\circ}$ que están cargadas de esfuerzos. Esta característica se debe a la interacción entre la zona de subducción y el campo de esfuerzos dentro de la zona de subducción, además esta característica se debe a una gran distribución de las asperezas propuestas por Beck y Ruff (1989) [Bec89].

\section{Evento sísmico de 2007 (8.0 Mw)}

El modelo de distribución y transferencia de esfuerzos de Coulomb para este sismo considera el modelo de Sladen et al. (2010) [Sla10]. Los resultados muestran una mayor amplitud en la relajación de los esfuerzos hacia el continente, esto implica un alto cambio cosísmico para homogeneizar los estados de los esfuerzos en el sismo. Esto se debe a que la zona de relajación tiene los valores de deslizamiento más altos y en menor amplitud en la dirección de la fosa peruana. La transferencia de esfuerzos en la ciudad de Pisco fue de -16.527 bar. Este resultado implicó una alta relajación de esfuerzos en la sub-falla donde se encontraba la ciudad de Pisco y un alto acoplamiento. La transferencia de esfuerzos en la ciudad de Cañete fue de $-0,478$ bar, lo que implicó una relajación de esfuerzos y un valor promedio de acoplamiento sísmico, además de una menor activación de la sismicidad (Figura 5).

\section{Conclusiones}

El análisis de la transferencia de esfuerzos estáticos de Coulomb ocurridos en la zona de subducción de la región central del Perú nos ha permitido llegar a las siguientes conclusiones:

Para el evento sísmico de 1940 (Mw 8.0), la propagación del incremento de los patrones lobulares de trnsferencia de esfuerzos de Coulomb se entendió hacia el norte de la ciudad de Huarmey, registrando valores de 0.568 bar que implicaban una menor activación sísmica, en la ciudad de Huacho, zona epicentro, la transferencia de tensiones se relajó tomando valores de -9.614 bares, esta relajación de tensiones se extendió al continente y a la fosa peruana. 
Para el evento sísmico de 1966 (Mw 8.1), la fuente sísmica se discretizó según Jiménez et al. (2016), se observa que la zona norte cerca de la costa de Huarmey permaneció cargada de tensiones porque la transferencia de esfuerzos de Coulomb tomó un valor de 0.382 bares, este resultado define una activación sísmica cerca de la costa de las ciudades de Huacho y Huarmey hacia el norte de la ciudad de Chimbote y hacia el sur a la ciudad de Lima, limitada entre la fosa peruana y la línea costera.

Para el evento sísmico de 1974 (8.1 Mw), los lóbulos de acumulación de esfuerzos se extienden a lo largo de los extremos norte y sur de la fuente sísmica, la Bahía de Pisco y las áreas circundantes tienen una alta acumulación de esfuerzos para liberar las costas de Huacho. Los lóbulos de relajación de esfuerzos están en dirección al continente y a la fosa peruana. La ciudad de Huacho tiene una concentración de esfuerzos de 0.144 bares, la ciudad de Lima tiene una concentración de esfuerzos de -4.187 bares, la ciudad de Cañete tiene una concentración de esfuerzos de -0.241 bares, en estas dos últimas áreas los esfuerzos han sido relajados. Finalmente, la ciudad de Pisco tiene una concentración de esfuerzos de 0.140 bares.

El modelo de distribución y transferencia de esfuerzos de Coulomb para el evento sísmico de 2007 (8.0 Mw) considera el modelo de Sladen et al., 2010 cuyos resultados muestran que, con mayor amplitud, la relajación de los esfuerzos en la dirección del continente implica un alto cambio cosísmico para homogeneizar los estados de los esfuerzos en el sismo, esto se debe al hecho de que esta zona de relajación tiene los valores de deslizamiento más altos y en menor amplitud en la dirección hacia la zanja. En la ciudad de Pisco hemos obtenido una transferencia de esfuerzos de -16.527 bares. Este resultado implicó una alta relajación de lo esfuerzos en el defecto por defecto correspondiente y un alto acoplamiento en la ciudad de $P$ isco. En la ciudad de Cañete hemos obtenido un valor de transferencia de esfuerzos de $-0,478$ bar que implica relajación de esfuerzos y un valor promedio de acoplamiento sísmico además de una menor activación de la sismicidad. El peligro de un evento sísmico futuro hasta la fecha de esta investigación está dentro de las costas del sur de Lima entre paralelos entre paralelos $-12^{\circ}$ y $-13^{\circ}$ y al sur de Pisco en las costas de la ciudad de Nazca entre la dorsal de Nazca y fosa peruana. En la bahía de Pisco, las tensiones han sido liberadas. La correlación de las réplicas con los lóbulos de tensión es considerablemente correcta, ya que se encuentra en los límites sur y norte del plano de falla.

Teniendo en cuenta los 4 sismos de subducción estudiados, aproximadamente el $20 \%$ de la energía total liberada por el sismo de 1746 fue liberada por la secuencia sísmica de 1940-2007 en la región central del Perú.

\section{Agradecimientos}

Esta investigación fue apoyada por CONCYTECFONDECYT en el marco de la convocatoria (Proyecto de investigación Básica 2015-I) [número de contrato 1002015]. Se agradece al Instituto Geofísico del Perú (IGP) por proporcionar todas las facilidades para desarrollar esta investigación durante la estadía de investigación.

\section{Referencias}

[Bar79] Barazangi, M y Isacks, B. (1979). Subduction of the Nazca plate beneath Peru: Evidence from the spatial distribution of earthquakes. Geoph. Jour. Roy. Astr. Soc., vol 57, pp 537-555.

[Bec89] Beck, S y Ruff, L. (1989). Great earthquakes and subduction along the Peru trench. Physics of the Earth and Planetary Interiors, 57, 199-224.

[Cah92] Cahill, Ty lsacks, B. (1992). Seismicity and shape of the subducted Nazca plate. J. Geophys. Res, vol 97, pp 17503-17529.

[Chl11] Chlieh, M., Perfettini, H., Tavera, H., Avouac, J.P., Remy, D., Nocquet, J. M., Rolandone, F., Bondoux, F., Gabalda, G., y Bonvalot, S. (2011), Interseismic coupling and seismic potential along the Central Andes subduction zone, J. Geophys. Res., 116, B12405, doi:10.1029/2010JB008166.

[Dor90] Dorbath, L., Cisternas, A y Dorbath, C. (1990).
Assesment of the size of large and great historical earthquakes in Peru. Bulletin of Seismological Society of America. Vol 80, No. 3, pp 551-576.

[Fre05] Freed A. (2005). Earthquake triggering by static, dynamic, and postseismic stress transfer. Annual Review of Earth and Planetary Sciences, vol. 33(1), pp 335-367.

[Gar06] Gardi, A., Lemoine, A., Madariaga, R y Campos, J. (2006). Modeling of stress in the Coquimbo region of central Chile. Journal of Geophysical Research, vol 111, doi:10.1029/2004JB003440.

[Has81] Hasegawa, A y Sacks, S. (1981). Subduction of Nazca plate beneath Peru as determined by seismic observations. J. Geophys. Res, vol 86, pp 4971-4980.

[Jim13] Jiménez, C., Moggiano, N., Mas, E., Adriano, B., Koshimura, S., Fuji, Y., Yanagisawa, H. (2013). Seismic source of 1746 Callao Earthquake from Tsunami Numerical Modeling. Journal of Disaster Research, vol 8, No.2, pp 266-273. 
[Jim15] Jiménez, C. (2015). El maremoto notable de 1746. Tesis de Maestría, Universidad Nacional Mayor de San Marcos, Lima-Perú.

[Jim16] Jiménez, C., Moggiano, N., Yauri, S., y Calvo, M. (2016). Fuente sísmica del terremoto de HuachoPerú 1966 de $8.1 \mathrm{Mw}$ a partir de inversión de registros mareográficos. Revista de Investigación de Física 19, 191601401.

[Kel72] Kelleher, J. (1972). Rupture zones of large South American earthquakes and some predictions. Journal of Geophysical Research, vol 77, No. 11, pp 20872103.

[Kin94] King, G., Ross, S., Lin, J. (1994). Static stress changes and the triggering of earthquakes. Bulletin of the Seismological Society of America, vol. 84, No. 3, pp 935-953.

[May16] Mayorga, E and Sánchez, J. (2016). Modelling of Coulomb stress changes during the great $(\mathrm{Mw}=8.8) 1906$ Colombia-Ecuador earthquake. Journal of South American Earth Sciences 70, pp 268-278. http://dx.doi.org/10.1016/j.jsames . 2016.05 .009

[Mia12] Miao, M and Zhu, S. (2012). A study of the impact of Static Coulomb Stress Changes of megathrust erthquakes along subduction zone on the following aftershocks. Chinese Journal of Geophysics, vol. 55, No.5, pp 539-551.

[Oka92] Okada, Y. (1992). Internal deformation due to shear and tensile faults in a half space. Bulletin of Seismological Society of America, vol. 82, No. 2, pp 1018-1040. 268-278.

[Rob01] Robinson, R., Benites, R., Ocola, L., Aleman, H. (2001). Stress transfer due to the June 23, 2001, Arequipa, Peru, Earthquake and previous large subduction events. American Geophysical Union, Fall Meeting, abstract id. S52A-0613
[Rod91] Rodriguez, L., Tavera, H. (1991). Determinación con alta resolución de la Geometría de la zon WadatiBenioff en el Perú Central. Revista Brasilera de Geofísica, vol 9, pp 141-159.

[Sch87] Schneider, J. y Sacks, I. (1987). Stress in the contorted Nazca plate beneath southern Peru from local earthquakes. Journal of Geophysical Research 92: doi: 10.1029/JB080i013p13887.

[Sla10] Sladen, A., Tavera, H., Simons, M., Avouac, P., Konca, O., Perfettini, H., Audin, L., Fielding, E., Ortega, F., y Cavagnoud, R. (2010). Source model of the $2007 \mathrm{Mw}$ 8.0 Pisco, Peru earthquake: Implications for seismogenic behavior of subduction megathrusts. Journal of Geophysical Research, Vol. 115, B02405, doi: 10.1029/2009JB006429.

[Ste99] Stein, R. (1999). The role of stress transfer in earthquake occurrence. Nature Vol. 402, pp. 605-609.

[Tav98] Tavera, H., Buforn, E. (1998). Sismicidad y Sismotectónica de Perú. Física de la Tierra, No. 10, pp 187-219.

[Tod11] Toda, S., Stein, R., Sevilgen, V., y Lin, J. (2011). Coulomb 3.3 Graphic-Rich deformation and stress-change software for earthquake, tectonic, and volcano research and teaching-user guide. Software manual: https://pubs.usgs.gov/of/2011/1060/ of 2011-1060.pdf

[Vil16] Villegas-Lanza, J., Chlieh, M., Cavalié, O., Tavera, H., Baby, P., Chire, J., y Nocquet, J. (2016). Active tectonics of Peru: Heterogeneous interseismic coupling along the Nazca megathrust, rigid motion of the Peruvian Sliver, and Subandean shortening accommodation. Journal of Geophysical Research: Solid Earth. doi: 10.1002/2016JB013080. 